\title{
Trans-urethral Ultrasound: A New Tool for Diagnostic and Therapeutic Imaging in Prostate Cancer
}

\author{
David R. Holmes III ${ }^{1}$, Brian J. Davis ${ }^{2}$, Richard A. Robb ${ }^{1}$ \\ ${ }^{1}$ Biomedical Imaging Resource and ${ }^{2}$ Division of Radiation Oncology \\ Mayo Foundation, Rochester, MN 55902
}

\begin{abstract}
We have developed a new imaging method for use in diagnosis and treatment of prostate cancer. Our laboratory is the first to publish ${ }^{1,2}$ this method that involves inserting into the urethra a special small diameter catheter with a phased array ultrasound imaging module on the tip which images the surrounding prostate gland in real time and three dimensions, capturing both anatomy and physiology. It is well tolerated by patients, as the imaging catheters are only 2 to $3 \mathrm{~mm}$ in diamter. Since there are nearly 200,000 new cases of prostate cancer diagnosed each year, improved methods for real-time, high-resolution 3D visualizations of the prostate are ever more important for accurate differential diagnosis and for staging optimal therapy.
\end{abstract}

Screening for prostate disease involves a digital rectal exam and a prostate specific antigen (PSA) test. If a patient tests positive, a tissue biopsy is collected and analyzed to classify and stage treatment of the disease. Depending on the characterization of the disease as benign or malignant, one or more treatment methods may be pursued. These include "watchful waiting", hormonal therapy, prostatectomy, radiation therapy (external beam or local brachytherapy), microwave ablation or cryotherapy. Imaging is an essential tool in each of these forms of treatment. However, diagnostic and therapeutic imaging of the prostate is challenging. The prostate gland is surrounded by the heavy pelvic girdle and lies medially among adjacent tissues and organs. Currently no imaging modality can distinguish diseased tissue from normal tissue in the prostate. Experimental diagnostic imaging techniques, such as elastography and color Doppler imaging have potential but require sophisticated image processing. Therapy guidance imaging includes intraoperative transrectal ultrasound (TRUS), $\mathrm{x}$ ray fluoroscopy and/or pre- and post-operative CT or MRI. Each of these modalities has advantages and disadvantages. Transurethral ultrasound (TUUS) imaging has potential to deliver many of the desired advantages with few disadvantages.

Imaging from the urethra has the obvious benefit of imaging from the center of the prostate. The reduced anatomic coverage (as compared to TRUS) allows higher frequency ultrasound to be used and consequently high-resolution images to be obtained. Therefore, TUUS images provide clear delineation of the prostate capsule, excellent tissue differentiation, improved contrast between tissue and radioactive seeds, and improved visualization of small structures, such as microvessels. In addition, TUUS imaging is inexpensive, real-time and both $2 \mathrm{D}$ and $3 \mathrm{D}$.

We have performed a number of preliminary experiments with TUUS imaging of the prostate. Comparisons between TUUS, TRUS and CT illustrated several favorable advantages of TUUS images. These were quantitatively demonstrated with a prostate

W. Niessen and M. Viergever (Eds.): MICCAI 2001, LNCS 2208, pp. 1243-1244, 2001.

(C) Springer-Verlag Berlin Heidelberg 2001 
phantom. TUUS provided much higher differentiation between the prostate and surrounding tissues, more accurate measurement of prostate volume, and more accurate measurement of seeds planted in the phantom, as noted in Table 1.

Initial animal studies (canines) demonstrated the viability and utility of TUUS as a unique real-time 3D imaging tool. Excellent differentiation between the prostate, rectum, bladder and neurovascular bundle was obtained. Color flow Doppler imaging with TUUS demonstrated potential for assessment of angiogenesis. Preliminary patient studies have demonstrated usefulness of TUUS for intraoperative imaging. The resolution of TUUS images in patients is superior to that of both CT and TRUS, providing clear differentiation between the prostate and surrounding tissue. Localization of implanted seeds in brachytherapy procedures was superior in TUUS compared to TRUS. TUUS was better than CT in determining total prostate volume. Figure 1 illustrates how TUUS images may be used in a brachytherapy application.

Table 1

\begin{tabular}{|l|l|l|}
\hline $\begin{array}{l}\text { Imaging } \\
\text { Modality }\end{array}$ & $\begin{array}{l}\text { Voxel size } \\
(\mathrm{mm})\end{array}$ & $\begin{array}{l}\text { Seed Size Measured } \\
(\text { True: } 0.8 \times 4.5 \mathrm{~mm})\end{array}$ \\
\hline TRUS & $.2 \times .2 \times 5$ & $1.2 \mathrm{~mm} \times 7.5 \mathrm{~mm}$ \\
\hline CT & $.35 \times .35 \times 3$ & $2.1 \mathrm{~mm} \times 8.4 \mathrm{~mm}$ \\
\hline TUUS & $.1 \times .1 \times .1$ & $.63 \mathrm{~mm} \times 4.4 \mathrm{~mm}$ \\
\hline
\end{tabular}

Figure 1: Real-time TUUS image (arrow) fused with 3D model segmented from MRI scan. Note clear delineation of radioactive seeds in TUUS image (2-D sector scan).

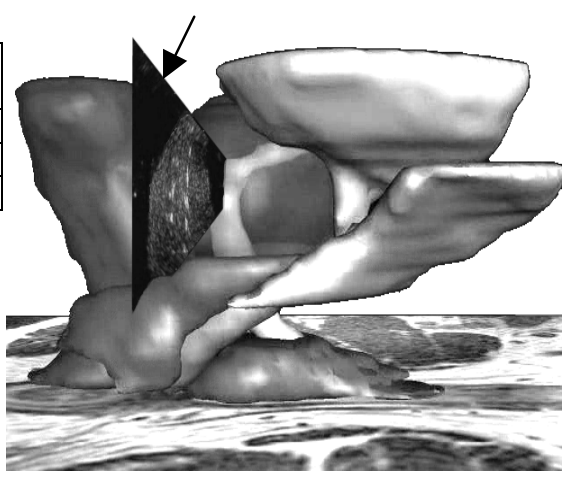

As a therapeutic tool, TUUS imaging has already been used in our institution during local brachytherapy procedures. Future TUUS devices will help to provide intraoperative feedback to the clinician during placement of radioactive seeds into the patient. Other treatments that might benefit from intraoperative TUUS include microwave therapy and cryoablation by monitoring the temperature of the prostate tissue during the procedure.

In conclusion, TUUS imaging has the potential to dramatically improve reliable detection and treatment of prostatic disease. It provides high-resolution, real-time 2D and 3D images for intra-operative applications, such as local brachytherapy. Utilizing ultrasound elastographic techniques with TUUS may provide a stiffness measure of the prostate. Doppler imaging with TUUS can help assess angiogenesis in the prostate. Further validation needs to be carried out, but preliminary results are very promising. Additional development will help provide systems and protocols for TUUS imaging in a variety of diagnostic and therapeutic scenarios.

1. Holmes DR and Robb RA. Trans-Urethral Ultrasound (TUUS) Imaging for Visualization and Analysis of the Prostate and Associated Tissues. Proc. Medicine Meets Virtual Reality. 70:126-132, 2000.

2. Holmes DR and Robb RA. 3-D Trans-urethral Ultrasound: a New View of the Prostate Gland. Computer Aided Radiology and Surgery, 2000. 